\title{
Primary malignant melanoma of the bronchus intermedius
}

\author{
Pier Luigi Filosso, MD, ${ }^{a}$ Giovanni Donati, MD, ${ }^{a}$ Enrico Ruffini, MD, ${ }^{a}$ Sabrina Croce, MD, ${ }^{\mathrm{b}}$ Mauro Papotti, MD,
}

Alberto Oliaro, $\mathrm{MD}^{\mathrm{a}}$ and Maurizio Mancuso, MD, ${ }^{\mathrm{a}}$ Torino, Italy

$\mathrm{M}$ elanoma is widely known as the most lethal of all skin cancers, and pulmonary metastases are the most common presentation of advanced disease. ${ }^{1}$ Melanoma of the eye, oral mucosa, genital and perineal areas, subungual and plantar surfaces, scalp, and palms are described, and although rare, they together make up about $10 \%$ of melanomas. ${ }^{2}$ Primary pulmonary melanoma is the rarest type of visceral melanoma: only 20 cases have been previously reported in the English literature. We describe the case of a patient in whom a primary melanoma of the bronchus was diagnosed and radically resected, and we discuss the cause and outcome of this rare pathology.

\section{Clinical Summary}

A 55-year-old white male smoker (40 pack-years) was referred to us for an endobronchial lesion discovered after complaints of chest pain, cough, and hemoptysis (Figure 1). Bronchoscopy revealed a large polylobulated lesion originating from the intermediate bronchus; histology at bronchial biopsy revealed a malignant melanoma. A preoperative brain and abdomen computed tomographic scan did not show any pathologic lesion. A thorough clinical examination of the body was performed by an experienced dermatologist: 2 suspect skin lesions (the first in the anterior left hemithorax and the second in the ipsilateral arm) were resected, but histology showed normal dermal nevi in both lesions. The patient underwent an accurate evaluation of other sites at which melanoma occasionally might occur: no oral and nasopharyngeal mucosa, anal and rectal mucosa, or eye lesions were detected. Furthermore, no family history for melanoma was documented. The patient underwent a right posterolateral thoracotomy, and a right pneumonectomy was performed; limited resection (sleeve) was not feasible because of tumor site and size and the high risk of positive margins (Figure 2). Mediastinal and hilar lymphadenectomy was performed. Grossly, the lesion was polilobulated and light red in color, measuring $3.2 \times 2 \mathrm{~cm}$ (Figure 3). The pleural surface, the lung, and all resected lymph nodes were free of tumor.

From the Departments of Thoracic Surgery and Oncology and Biomedical Sciences, University of Torino, Italy, San Giovanni Battista Hospital, ${ }^{\mathrm{b}}$ Torino, Italy.

Received for publication Nov 5, 2002; revisions requested Nov 21, 2002; revisions received Dec 25, 2002; accepted for publication Dec 27, 2002.

Address for reprints: Pier Luigi Filosso, MD, University of Torino, Italy, San Giovanni Battista Hospital, Department of Thoracic Surgery, Via Genova, 3 10126, Torino, Italy (E-mail: pierluigifilosso@tiscalinet.it).

J Thorac Cardiovasc Surg 2003;126:1215-7

Copyright $(2003$ by The American Association for Thoracic Surgery $0022-5223 / 2003 \$ 30.00+0$

doi:10.1016/S0022-5223(03)00691-3

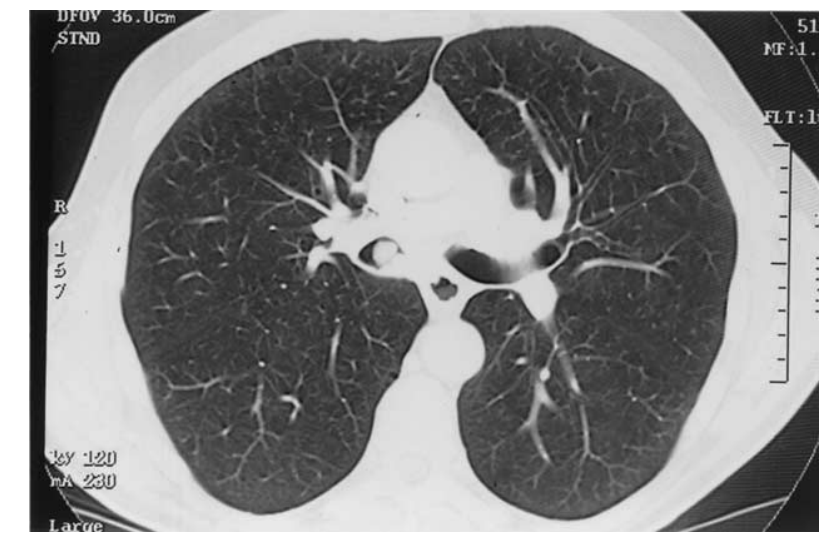

Figure 1. Thoracic computed tomographic scan showing an endobronchial lesion of the bronchus intermedius.

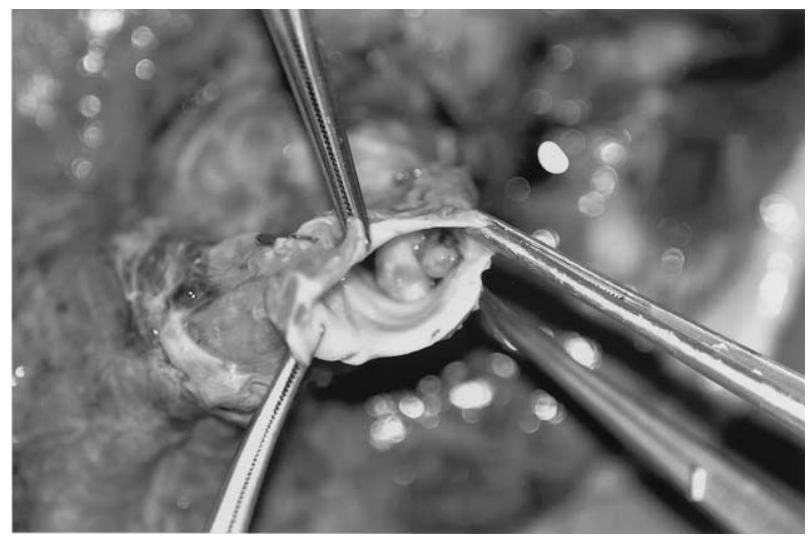

Figure 2. Gross specimen of the resected right lung with an endobronchial neoplastic lesion, which originates within the bronchus intermedius.

Histologically, the tumor was submucosal, without involvement of the bronchial cartilage. The tumor grew in a lobar pattern, and the neoplastic cells showed an epithelioid feature, with large cytoplasm, giant nuclei, prominent nucleoli, and numerous mitotic figures (Figure 4). Some cells showed cytoplasmic granular brown pigment. Immunohistochemistry showed strongly positive cytoplasmic staining of tumor cells with antibodies to S-100 protein (polyclonal antibody, 1:3500; Dako, Glostrup, Denmark) and HMB-45 (1:30; Biogenex, San Ramon, Calif; Figure 5). No staining was observed in the bronchial mucosa, and a diagnosis of malignant melanoma was thus confirmed (Table 1).

The postoperative course was uneventful, and the patient was discharged from the hospital in good general condition. Adjuvant 


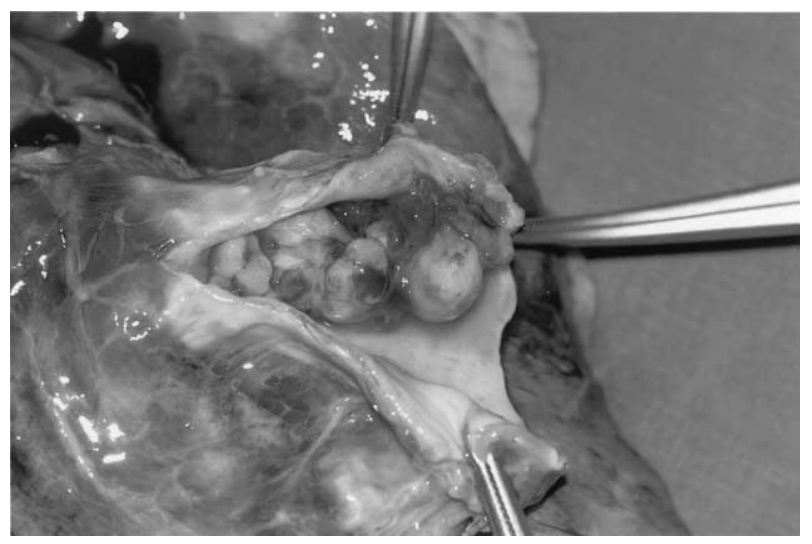

Figure 3. Gross specimen of the endobronchial malignant melanoma.



Figure 4. Malignant bronchial melanoma: note the epithelioid feature of the neoplastic cells, with large cytoplasm and giant nuclei. (Hematoxylin and eosin, original magnification $40 \times$.)

chemotherapy was scheduled, and the patient is alive and free of disease 14 months after the treatment.

\section{Discussion}

Primary pulmonary melanoma is exceedingly rare: only 20 cases have been previously reported in the English-language literature. ${ }^{3}$ Malignant melanoma represents the most common cause of death caused by skin neoplasm; in case of advanced disease, the lung is a common site of distant metastases. When the lung is involved, a metastatic pattern appears as a solitary pulmonary nodule, a possible cause of misinterpretation as a primary lung cancer. Allen and Drash ${ }^{4}$ proposed 3 criteria to distinguish primary pulmonary melanoma from metastatic disease: junctional change with nesting of malignant cells beneath the bronchial epithelium, invasion of the bronchial epithelium in an area without epithelial ulceration, and demonstration of melanoma beneath the aforementioned changes. A correct diagnosis of primary pulmonary melanoma requires a thorough clinical evaluation of the patient to rule out occult sites of melanoma.

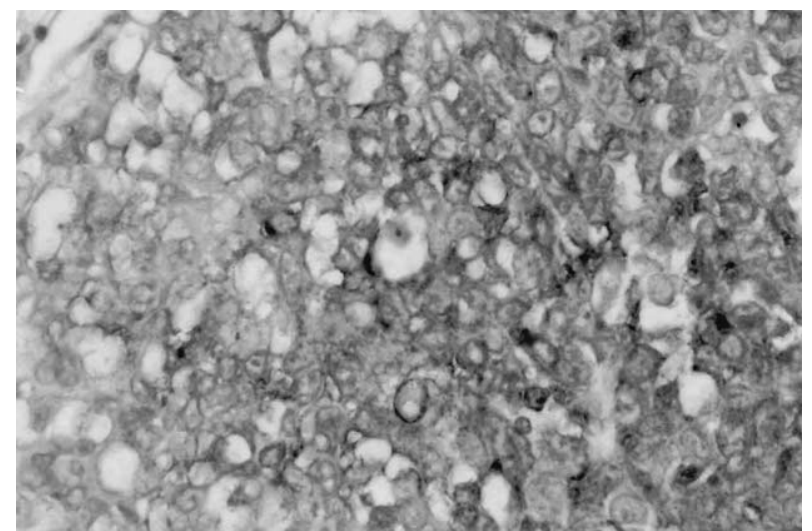

Figure 5. Strongly positive cytoplasmic staining of malignant cells with antibody to HMB-45 (1:30).

Accurate skin, eye, nasopharyngeal and oral mucosa, and anorectal mucosa examination is mandatory because these sites are occasionally the source of occult melanomas. Jensen and Egedorf ${ }^{5}$ proposed clinical criteria for the diagnosis of primary pulmonary melanoma: no previously removed pigmental skin tumors; no demonstrable melanomas in other organs at surgical intervention because the pulmonary lesion might represent a metastatic disease; no history of resected ocular tumors; solitary pulmonary tumor, with morphologic features compatible with those of a primary lung tumor; and no other primary melanomas in cases in which autopsy is available.

In the present case melanoma was not demonstrable either in the skin or in other unusual sites after a thorough clinical examination. In addition, the gross aspect of the lesion was compatible with an endobronchial primary lung cancer.

The development of a primary melanoma within the bronchial tree remains controversial. Authors have hypothesized that pulmonary melanoma could arise from a small number of melanoblasts because both the pharynx and esophagus can give rise to primary melanomas and because the esophagus and trachea have a common embryologic origin. ${ }^{5-8}$

Therefore the possibility that a malignant bronchial lung melanoma is a metastasis of a regressed skin melanoma is to be considered. In fact, malignant melanoma of the skin might spontaneously regress, becoming clinically unapparent and yet having metastasized. ${ }^{9}$ Our patient had no clinically proven suspect or resected skin lesions before the diagnosis of endobronchial melanoma, and no familiarity for melanoma was documented. The most common complaints of primary pulmonary melanoma described in the previously reported 20 patients are cough (19\%), hemoptysis (40\%), postobstructive pneumonia (25\%), lobar collapse or atelectasis $(25 \%)$, and an incidental finding on chest radiography $(30 \%)$. Our patient presented with chest pain, cough, and hemoptysis.

The patient was 55 years old, which is in accordance with the mean age reported in the literature. He was white and without a family history of melanoma. All the patients previously described were white, and family history was documented in only 1 patient. Our patient was a 40 pack-year cigarette smoker: of the 20 previous patients, 6 were smokers, 5 were nonsmokers, and 9 had 
TABLE 1. Differentiation between a primary melanoma of the lung and a melanin-containing carcinoid according to ultrastructural and immunohistochemical features

\begin{tabular}{|c|c|c|}
\hline Primary melanoma & & $\begin{array}{l}\text { Melanin-containing } \\
\text { carcinoid }\end{array}$ \\
\hline \multicolumn{3}{|l|}{ Ultrastructural features } \\
\hline Cytoplasmic organelles & & $\begin{array}{l}\text { Abundant } \\
\text { mitrochrondia }\end{array}$ \\
\hline $\begin{array}{l}\text { Smooth endoplasmatic } \\
\text { reticulum }\end{array}$ & & Desmosomes \\
\hline $\begin{array}{l}\text { No neurosecretory } \\
\text { granules }\end{array}$ & & $\begin{array}{l}\text { Neurosecretory } \\
\text { granules }\end{array}$ \\
\hline \multicolumn{3}{|l|}{$\begin{array}{l}\text { Immunohistochemical } \\
\text { features }\end{array}$} \\
\hline+++ & S-100 protein & \pm \\
\hline- & NSE & +++ \\
\hline- & Calcitonin & ++ \\
\hline- & Keratin & + \\
\hline- & Ep Memb Antigen & + \\
\hline- & Chromogranin A & ++ \\
\hline
\end{tabular}

NSE, Neuron-specific enolase; Ep Memb Antigen, epithelial membrane antigen.

undocumented smoking status. ${ }^{3}$ Endobronchial location of the tumor is common; as Ost and colleagues ${ }^{3}$ report, in 13 patients in whom bronchoscopy was performed, tumor detection was possible in $8(61.5 \%)$. In these patients the tumor was described as black or pigmented in 4 patients and nonpigmented in 4 patients. Our patient had a polilobulated nonpigmented lesion of the intermediate bronchus, and preoperative biopsy was effective in diagnosing melanoma. No data have been previously reported in the literature about the effectiveness of bronchial biopsy in a correct preoperative diagnosis of the endobronchial melanoma. In some cases bronchial biopsy detects a non-small cell carcinoma; correct diagnosis was obtained after the operation. Only 15 surgical procedures were performed in 20 patients, according to Ost and colleagues $^{3}$ review of the literature: 5 pneumonectomies, 8 lobectomies, and 2 segmentectomies. We were obliged to perform a right pneumonectomy because the site of the tumor did not allow any other resection.

The presence of lymph node involvement at the time of the operation did not preclude long-term survival. Ost and colleagues ${ }^{3}$ report that of 15 patients who underwent surgical resection of the tumor, $6(40 \%)$ had lymph node involvement documented at the time of the operation. Of these patients, 4 experienced recurrent disease, and 2 were free of disease at 3 and 11 years, respectively. Our patient underwent a hilar and mediastinal lymphadenectomy: only subcarinal lymph nodes were grossly large, black, and suspect for metastases, but this hypothesis was not confirmed at definitive histology.

In conclusion, the success of a radical resection with long-term survival in 5 patients (median survival, 30 months), ${ }^{3}$ suggest that an aggressive surgical approach is mandatory in primary pulmonary melanoma. Anatomic resection is considered the treatment of choice because segmentectomy can be complicated with recurrence and with poor survival. Hilar and mediastinal lymphadenectomy is mandatory. We suggest the utility of an adjuvant chemotherapeutic treatment to prevent recurrences or distant metastases.

\section{References}

1. Gromet MA, Ominsky SH, Epstein WL. The thorax as the initial site for systemic relapse in malignant melanoma. Cancer. 1979;44:776-84.

2. Rogers RS III, Gibson LE. Mucosal, genital and unusual clinical variants of melanoma. Mayo Clin Proc. 1997;72:326-66.

3. Ost D, Joseph C, Sogoloff H, Menezes G. Primary pulmonary melanoma: case report and literature review. Mayo Clin Proc. 1999;74:62-6.

4. Allen MS Jr, Drash EC. Primary melanoma of the lung. Cancer. 1968;21:154-9.

5. Jensen OA, Egedorf J. Primary malignant melanoma of the lung. Scand J Respir Dis. 1967;48:127-35.

6. Batsakis JG, Regezi JA, Solomon AR, Rice DH. The pathology of head and neck tumors: mucosal melanomas, part 13. Head Neck Surg. 1982; 4:404-18.

7. Robertson AJ, Sinclair DJ, Sutton PP, Guthrie W. Primary melanocarcinoma of the lower respiratory tract. Thorax. 1980;35:158-9.

8. Carstens PH, Kuhns JG, Ghazi C. Primary malignant melanoma of the lung and adrenal. Hum Pathol. 1984;15:910-4.

9. Smith JL, Stehlin JS Jr. Spontaneous regression of primary malignant melanoma with regional metastases. Cancer. 1965;18:1399-415. 